\title{
In-line System to Produce High-Purity Acid Solutions
}

\author{
Hiroto Masunaga, *广 Yuji Higo,* Mizuo IshiI,* Noboru Maruyama,* and Shigeo Yamazaki** \\ *Nichiri Mfg. Co., Ltd., 324-5 Takatsu, Yachiyo, Chiba 276-0036, Japan \\ **Department of Applied Science, Okayama University of Science, 1-1 Ridaicho, Okayama 700-0005, Japan
}

\begin{abstract}
Herein, we report a new device that generates a high-purity acid solution. It comprises three compartments divided by anion-exchange membranes and filled with ion-exchange resins. Fluorochemical cation-exchange membranes, which tolerate electrochemical wear and permit bulk flow, are inserted between each electrode and the anion-exchange resin. A bipolar boundary is a composite boundary comprising anion and cation exchangers. This device has four bipolar boundaries to separate the location of acid generation from the location where water is electrolyzed. It can tolerate high pressures, resist degradation due to electrolysis at the electrodes, and produce high-purity acid solutions that are free from gases and cationic impurities. The acid solution is generated on the basis of an electrokinetic phenomenon at the surfaces of ion-exchange resins and membranes in an electric field; its concentration can be controlled at rates from 0.01 to $100 \mu \mathrm{mol} / \mathrm{min}$ by adjusting the electrical current applied to the device.
\end{abstract}

Keywords Ion chromatography, cation analysis, acid solution generation, electrokinetic phenomenon, separation of acid solution generation place from water electrolysis place, fluorochemical cation exchanger

(Received December 2, 2015; Accepted February 24, 2016; Published June 10, 2016)

\section{Introduction}

Acid solutions have been widely used in analytical techniques employed by scientists and engineers. They have been used as carriers, eluents, and diluents for techniques, such as HPLC, LC. In all applications, the acid solutions have been prepared and replenished by the user. This paper specifically addresses the production of methanesulfonic acid (MSA) and $\mathrm{HCl}$ solutions. Conventional acid solution preparation methods involve dilution of concentrated acid; the resulting solutions contain varying amounts of dissolved cations, such as $\mathrm{Na}^{+}$, $\mathrm{NH}_{4}^{+}$, and $\mathrm{K}^{+}$. Acid purification involves removing these cations, which is extremely difficult, time consuming, and often tedious. ${ }^{1}$ Therefore, automated methods for acid preparation are desirable. Dasgupta et al. ${ }^{2,3}$ pioneered the application of electrolytic methods to the generation of eluents for ion chromatography (IC) and demonstrated electrical control of concentration. Small et al. ${ }^{4,5}$ introduced a new method called ion reflux, wherein eluent generation and suppression were integrated into an automated form of IC that has the potential to continuously operate. Dionex introduced the EG40 eluent generator, ${ }^{6-8}$ which uses one of two cartridges to produce either a $\mathrm{KOH}$ solution (for anion analysis) with a cation exchanger or MSA (for cation analysis) with an anion exchanger. The eluent's concentration is controlled by the amount of current applied to the ion exchange unit. The device cartridges have a dead volume of less than $300 \mu \mathrm{L}$. Since this device generates MSA solutions by the reaction of methanesulfonate ions (MS', arriving at the anode) with $\mathrm{H}^{+}$(generated by electrolysis of

† To whom correspondence should be addressed.

E-mail: h.masunaga@ nichiri-mfg.co.jp water on the anode), the generated MSA solution is pumped into the degas module inlet to remove the byproduct of electrolysis - $\mathrm{O}_{2}$ gas - at the anode. Therefore, the dead volume between the device and the separation column is increased..$^{8,9}$ The strong anion exchanger is known to undergo Hofmann elimination by attack of the hydroxyl group on the quaternary amine, and to be electrolyzed by oxidation and reduction at the electrodes. ${ }^{10}$

This study aims to develop a new device that can tolerate high pressures, resist degradation due to electrolysis at the electrodes, and produce a continuous stream of high-purity acid solution in line, which does not contain gases $\left(\mathrm{H}_{2}\right.$ or $\left.\mathrm{O}_{2}\right)$, produced by electrolysis of water at the electrodes and impurity cations. The device is schematically displayed in Fig. 1. It comprises three compartments that are isolated from each other by two anion-exchange (AE) membranes, and electrodes are placed at the far ends of the outside compartments. The three compartments are filled with ion-exchange resins, and the fluorochemical cation exchange membrane (FCEM), which permits bulk flow, is installed between each electrode and its adjacent $\mathrm{AE}$ resin. A center compartment contains both $\mathrm{AE}$ and cation exchange (CE) layers packed with $\mathrm{AE}$ and $\mathrm{CE}$ resins, respectively. A bipolar boundary is a composite boundary comprising an $\mathrm{AE}$ resin and a $\mathrm{CE}$ membrane, an $\mathrm{AE}$ resin and a $\mathrm{CE}$ resin, or an $\mathrm{AE}$ membrane and a $\mathrm{CE}$ resin. We anticipate that the bipolar boundary will show properties that are analogous to a bipolar membrane. The current-voltage $(I-V)$ properties of the bipolar boundary show the following rectification properties: A) a rapid increase of the electric current with the applied voltage under forward bias $(V>0$ in Fig. 2, where $V$ is the applied voltage) results in ions (anions and cations) becoming concentrated at the bipolar boundary (hereinafter referred to as the ion concentration junction, ICJ) and B) a region of high 


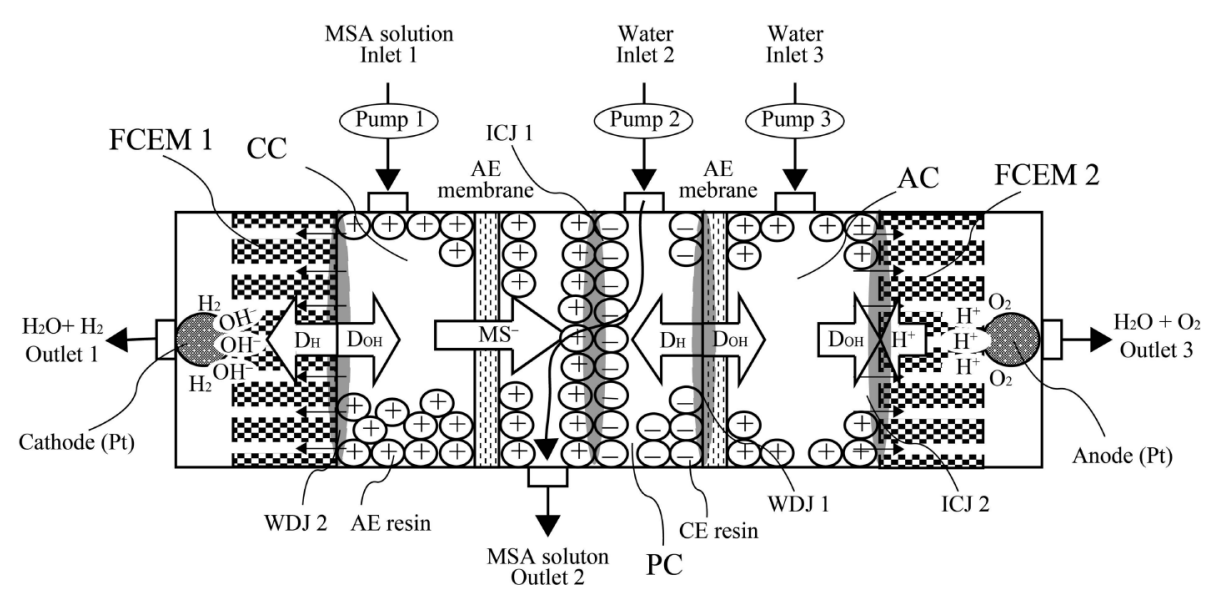

Fig. 1 Schematic of the four-bipolar-boundary device.

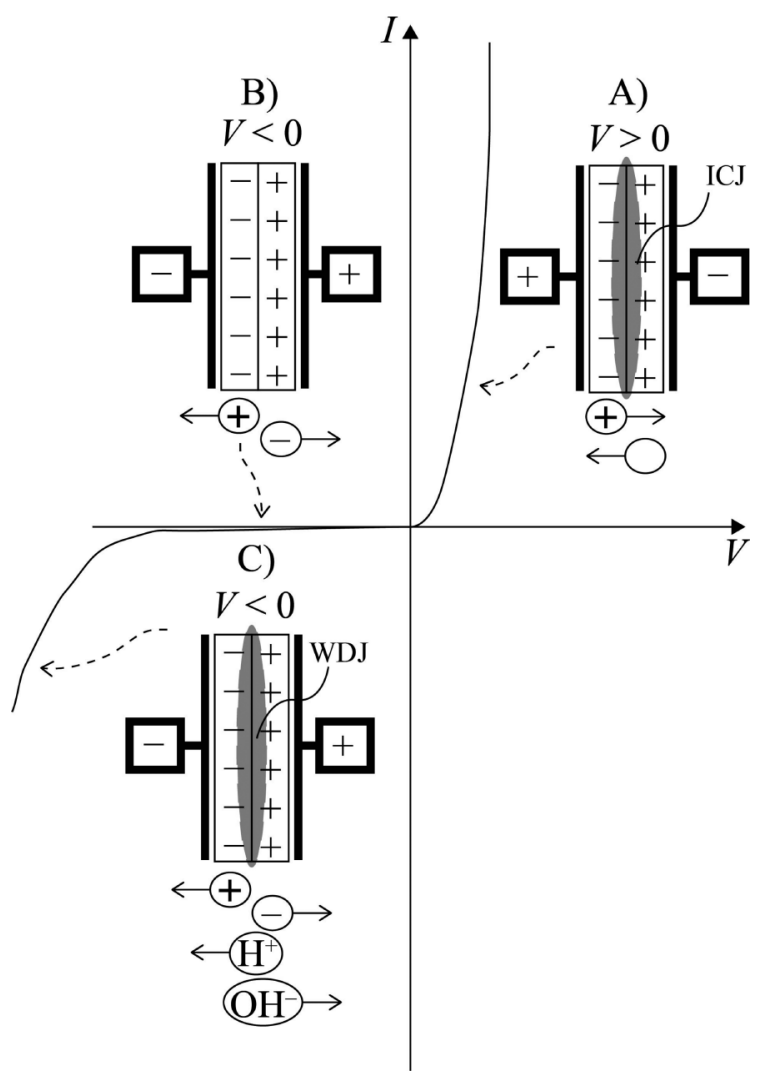

Fig. $2 I-V$ properties of the bipolar boundary. A) $V>0$, a region of ion concentration, B) $V<0$, low reverse bias voltages, a region of high resistance, and C) $V<0$, high reverse bias voltages, a region of water dissociation.

resistance when the membrane is reverse biased $(V<0$, low reverse-bias voltages) can be seen in Fig. 2.11,12 Under high reverse bias voltages, however, C) a rapid increase in current with the applied voltage $(V<0$ in Fig. 2 , high reverse bias voltages) due to an anomalously high water dissociation rate is observed (hereinafter referred to as the water-dissociation junction, WDJ). It is widely accepted that the large electric field occurring at the WDJ under reverse bias enhances the water dissociation rate via protonation-deprotonation reactions involving the ion-exchanger and the water molecules in the
WDJ. ${ }^{12-18}$ This device has four bipolar boundaries (ICJs 1 and 2 and WDJs 1 and 2). Consequently, the MSA (or $\mathrm{HCl}$ ) solution is generated at a separate position from where the water is electrolyzed. These specifications enable the four-bipolarboundary device to tolerate high pressures, resist degradation from electrolysis on the electrodes, and produce a high-purity MSA (or $\mathrm{HCl}$ ) solution that is free from gases and cationic impurities. This paper introduces the four-bipolar-boundary device developed for in-line MSA $(\mathrm{HCl})$ generation and examines the mechanism of its operation. An MSA (or $\mathrm{HCl}$ ) solution is generated by an applied current $(0-200 \mathrm{~mA})$ and the resulting concentration varies at rates from 0.01 to $100 \mu \mathrm{mol} /$ $\min$.

\section{Experimental}

Architecture of the four-bipolar-boundary device and comparison device

The four-bipolar-boundary device architecture is schematically displayed in Fig. 1. The device comprises three compartments isolated from each other by two AE membranes $(\phi 12 \mathrm{~mm}$, AHA, Astom, Tokyo). An electrode is placed at the far ends of the two outside compartments. The cathode compartment (CC, $15 \mathrm{~mm}$ in length) is packed with $\mathrm{AE}$ resin (Amberlite IRA402BL, $0.6-0.8 \mathrm{~mm}, \geq 1.25 \mathrm{meq} / \mathrm{mL}$, Organo, Tokyo, Japan) and FCEM 1 (CMF, physically modified to increase water permeability, $\phi 12 \mathrm{~mm}$, Asahi Glass, Tokyo) is inserted between the cathode and the AE resin. WDJ 2 consists of FCEM 1 and AE resin. The preparation compartment (PC, $15 \mathrm{~mm}$ in length) consists of both CE (Dowex 5WX8, $200-400 \mathrm{mesh}, 1.7 \mathrm{meq} / \mathrm{mL}$ ) and $\mathrm{AE}$ resins (Dowex 1X8, $200-400 \mathrm{mesh}, 1.2 \mathrm{meq} / \mathrm{mL}$ ). ICJ 1 consists of both AE and $\mathrm{CE}$ resins. The anode compartment (AC, $15 \mathrm{~mm}$ in length) is filled with AE resin (Amberlite IRA402BLB) and FCEM 2 $(\mathrm{CMF})$ is installed between the cathode and AE resin. WDJ 1 consists of CE resin and an AE membrane. ICJ 2 consists of AE resin and FCEM 2. There is bulk flow through FCEMs 1 and 2. Inlets 1,2 and 3 are connected to Pump 1 (peristaltic pump, with a $1 \mathrm{~mL} / \mathrm{min}$ flow rate, controlled by a home-made flow control driver), Pump 2 (1.3 mL/min, DP-8020, Tosoh, Tokyo, Japan) and Pump 3 (peristaltic pump, with a $1 \mathrm{~mL} / \mathrm{min}$ flow rate, controlled by a home-made flow control driver), respectively. A $100-\mathrm{mM}$ MSA or a $200-\mathrm{mM} \mathrm{NaCl}$ solution is pumped into Inlet 1, pure water is pumped into Inlets 2 and 3, 


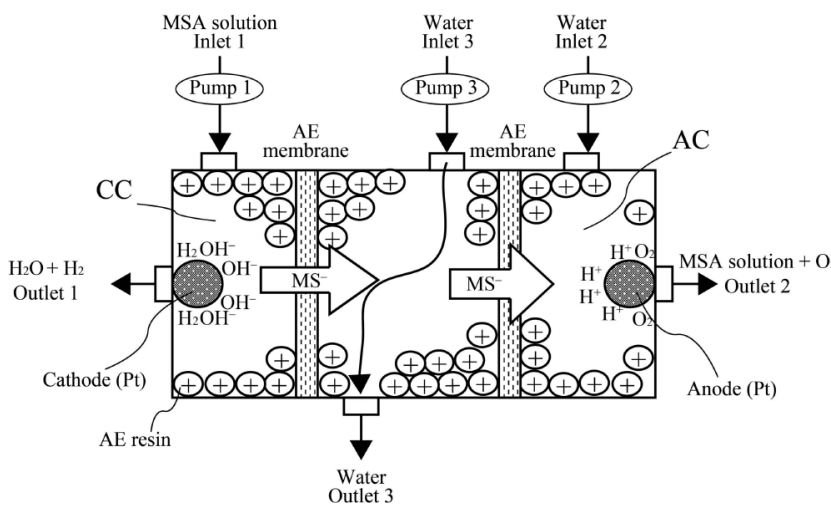

Fig. 3 Schematic of $\mathrm{CD}_{\text {boundary0. }}$.

and the purified MSA (or $\mathrm{HCl}$ ) solution is eluted from Outlet 2 of the PC.

A comparison device without a bipolar boundary, the $\mathrm{CD}_{\text {boundary } 0}{ }^{6-8}$ was built to test the proposed mechanisms of the four-bipolar-boundary device; this is schematically displayed in Fig. 3. $\mathrm{CD}_{\text {boundary0 }}$ is packed with only $\mathrm{AE}$ resin between the electrodes, so there is no bipolar-boundary between the electrodes. A $100-\mathrm{mM}$ MSA or a $200-\mathrm{mM} \mathrm{NaCl}$ solution is pumped into Inlet 1, pure water is pumped into Inlets 2 and 3, and the purified MSA (or $\mathrm{HCl}$ ) solution is eluted from Outlet 2 of the AC.

Electrical conductivity measurement of the MSA solution produced by the four-bipolar-boundary device and $C D_{\text {boundary }}$

The system used herein is schematically displayed in Fig. 4. Outlet 2 of the four-bipolar-boundary device and $\mathrm{CD}_{\text {boundary0 }}$ was connected to an electrical conductivity detector (ECD, iCM8401, Nichiri, Japan) to measure the electrical conductivity of the effluent streams.

Ion chromatograph equipped with the four-bipolar-boundary device

An ion chromatograph (ICA-2000) was equipped with a pump (1 mL/min, ICA-2000), a four-bipolar-boundary device, an injector $(20 \mu \mathrm{L}$, ICA-2000), a separation column for cation analysis (IonPac $\mathrm{CS} 14,40^{\circ} \mathrm{C}$, Dionex, USA), a suppressor (iSC8010, ${ }^{18}$ Nichiri), and an electrochemical detector (ECD, ICA-2000). The four-bipolar-boundary device was connected between the pump and the injector. Effluent $(\sim 10 \mathrm{mM}$ MSA solution, $3861 \mu \mathrm{S} / \mathrm{cm}, \mathrm{pH} 2.04)$ from Outlet 2 of the four-bipolar-boundary device was used as an eluent for IC. The injected sample was separated using the column, $\mathrm{MS}^{-}$was removed from the eluent using the suppressor, and the suppressor effluent was characterized using the ECD.

\section{Results and Discussion}

\section{Reaction mechanisms}

Ion-exchange resins and membranes have mobile counter ions and are good electronic conductors. ${ }^{19}$ We consider $\mathrm{CD}_{\text {boundary }}$, which retains only an anion exchanger between two metal electrodes in a chamber. When a DC potential is applied across the electrodes, the electrical field drives the negative counter ions toward the anode. ${ }^{4,5}$

In the case of our proposed four-bipolar-boundary device, when a DC potential is applied across the electrodes, we

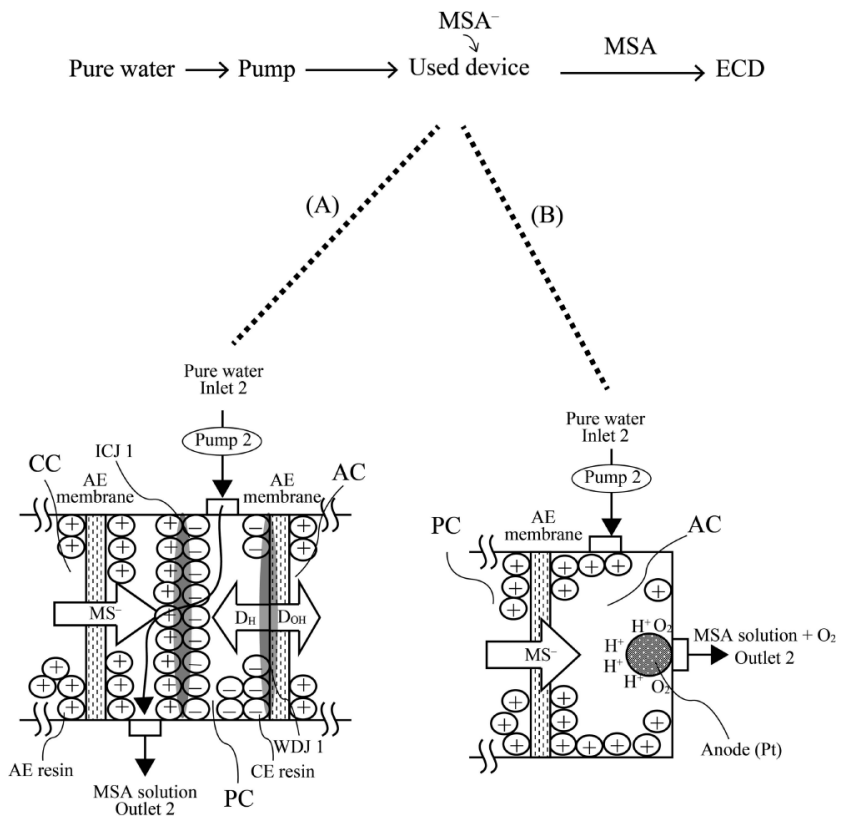

Fig. 4 Schematic of system used in this study. (A) The four-bipolarboundary device, and (B) the $\mathrm{CD}_{\text {boundary0 }}$.

anticipate that the following reactions occur at the four bipolar boundaries in addition to the oxidation-reduction reaction at the electrodes. The cathodic reduction of water produces $\mathrm{OH}^{-}$and $\mathrm{H}_{2}$ gas:

$$
2 \mathrm{H}_{2} \mathrm{O}+2 \mathrm{e}^{-} \longrightarrow 2 \mathrm{OH}^{-}+\mathrm{H}_{2}(\mathrm{~g})
$$

The anodic oxidation of water produces $\mathrm{O}_{2}$ gas and $\mathrm{H}^{+}$:

$$
2 \mathrm{H}_{2} \mathrm{O} \longrightarrow 4 \mathrm{H}^{+}+\mathrm{O}_{2}(\mathrm{~g})+4 \mathrm{e}^{-}
$$

Water dissociation produces $\mathrm{OH}^{-}$and $\mathrm{H}^{+}$at WDJs 1 and $2\left(\mathrm{OH}^{-}\right.$ and $\mathrm{H}^{+}$produced by water dissociation are hereinafter referred to as $\mathrm{D}_{\mathrm{OH}}$ and $\mathrm{D}_{\mathrm{H}}$, respectively):

$$
\mathrm{H}_{2} \mathrm{O} \longrightarrow \mathrm{D}_{\mathrm{H}}+\mathrm{D}_{\mathrm{OH}}
$$

Ion concentration produces MSA solution at ICJ 1:

$$
\mathrm{MS}^{-}+\mathrm{H}^{+} \longrightarrow \text { MSA solution }
$$

Ion concentration also produces $\mathrm{H}_{2} \mathrm{O}$ at ICJ 2:

$$
\mathrm{H}^{+}+\mathrm{D}_{\mathrm{OH}} \longrightarrow \mathrm{H}_{2} \mathrm{O}
$$

$\mathrm{D}_{\mathrm{H}}$ and $\mathrm{D}_{\mathrm{OH}}$ are produced by water dissociation at WDJ 2. $\mathrm{D}_{\mathrm{H}}$ migrates through FCEM 1 to the cathode and reacts with the $\mathrm{OH}^{-}$generated by electrolysis of water at the cathode. The byproduct of electrolysis at the cathode $-\mathrm{H}_{2}$ gas -is eluted out with the MSA solution stream passing from Inlet 1 to Outlet 1 , because the $\mathrm{CC}$ is separated from the $\mathrm{PC}$ by the $\mathrm{AE}$ membrane. Trace amounts of $\mathrm{NH}_{4}{ }^{+}$in the MSA solution are also eluted out with the stream. $\mathrm{D}_{\mathrm{OH}}$ sequentially migrates into the $\mathrm{AE}$ resin in the $\mathrm{PC}$, displacing other anions $\left(\mathrm{OH}^{-}\right.$and $\left.\mathrm{MS}^{-}\right)$. Therefore, $\mathrm{OH}^{-}$ and the $\mathrm{MS}^{-}$propagate a net motion toward the anode. The PC has two bipolar boundaries (ICJ 1 and WDJ 1); ICJ 1 comprises the $\mathrm{CE}$ and $\mathrm{AE}$ resins, and WDJ 1 consists of an $\mathrm{AE}$ membrane and $C E$ resin. $D_{H}$ and $D_{\mathrm{OH}}$ are also generated by water 


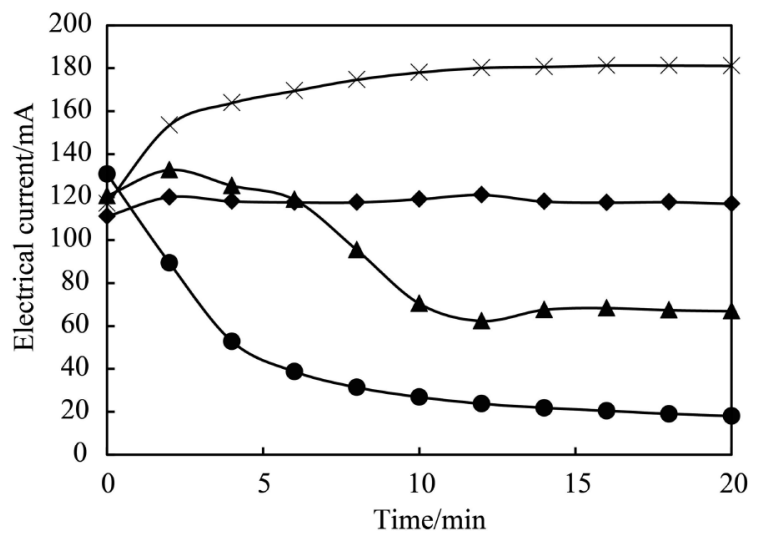

Fig. 5 Variation in the applied electrical current as a function of time for a constant potential of $30 \mathrm{~V}$ in the four-bipolar-boundary device: (ム) water is withheld from the PC, $(\times)$ water is withheld from the AC, $(\bullet)$ water is withheld from the $\mathrm{CC}$, and $(\bullet)$ water is pumped to all compartments (PC, AC, and CC).

dissociation at WDJ 1. $\mathrm{D}_{\mathrm{H}}$ and $\mathrm{D}_{\mathrm{OH}}$ migrate toward ICJ 1 and the anode, respectively. $\mathrm{CE}$ resin is a negatively charged stationary phase; anions, such as $\mathrm{OH}^{-}, \mathrm{Cl}^{-}$and $\mathrm{MS}^{-}$collect and concentrate at ICJ 1 because they cannot migrate to the anode over the $\mathrm{CE}$ resin. $\mathrm{AE}$ resin is a positively charged stationary phase; cations, such as $\mathrm{H}^{+}, \mathrm{Na}^{+}$, and $\mathrm{K}^{+}$also collect and concentrate at ICJ 1 because they cannot migrate to the cathode over the $\mathrm{AE}$ resin. If water is depleted in the $\mathrm{CC}$ and/or the PC, the current will not pass between the electrodes because there is no means for replenishing the population of $\mathrm{D}_{\mathrm{H}}$, or $\mathrm{D}_{\mathrm{OH}}$. Consequently, water has to be pumped into the $\mathrm{CC}$ and $\mathrm{PC}$ to maintain a constant electrical current between the electrodes. An MSA solution (or an $\mathrm{HCl}$ solution) is generated by the reaction between the $\mathrm{MS}^{-}$and $\mathrm{D}_{\mathrm{H}}\left(\right.$ or $\mathrm{Cl}^{-}$and $\mathrm{D}_{\mathrm{H}}$ ) concentrated at ICJ 1, and eluted out with the pure water stream passing from Inlet 2 to Outlet 2 across ICJ 1, because the PC is separated from the $\mathrm{AC}$ and the $\mathrm{CC}$ by two $\mathrm{AE}$ membranes. $\mathrm{H}^{+}$, which is generated by the electrolysis of water at the anode, migrates through FCEM 2 to the AE resin in the AC. The molar quantity of $\mathrm{H}^{+}$is identical to that of the $\mathrm{D}_{\mathrm{OH}}$ arriving at FCEM 2. The byproduct of electrolysis at the anode $-\mathrm{O}_{2}$ gas-is eluted out with the pure water stream passing from Inlet 3 to Outlet 3 because the $\mathrm{AC}$ is separated from the $\mathrm{PC}$ by the AE membrane. Cationic impurities and anions in the water are also eluted out with the stream. In the four-bipolar-boundary device in which electrochemical endurance is improved by the FCEM installed between each electrode and its adjacent $\mathrm{AE}$ resin, the acid solution is generated at ICJ 1 , which is separate from where the water is electrolyzed. Thus, high-purity acid solutions can be prepared in-line and are free from gases and cationic impurities.

Location of water dissociation at WDJs 1 and 2 and ion concentration at ICJS 1 and 2

We determined that $\mathrm{D}_{\mathrm{H}}$ and $\mathrm{D}_{\mathrm{OH}}$ are produced by the water dissociation at the WDJs and that ions are concentrated at the ICJs. If water is not continuously pumped into the $\mathrm{PC}$ and $\mathrm{CC}$ of the four-bipolar-boundary device, the water in the PC and CC is consumed by generation of $\mathrm{D}_{\mathrm{H}}$ and $\mathrm{D}_{\mathrm{OH}}$ at the WDJs. Thus the electrical current passing between the electrodes will decrease. If water is not pumped into the $\mathrm{AC}$ or $\mathrm{PC}$, ions are concentrated at ICJs 1 and 2. Thus, the electrical current between the electrodes will increase due to reduction of the electrical resistance at the ICJs. The variation in the electrical

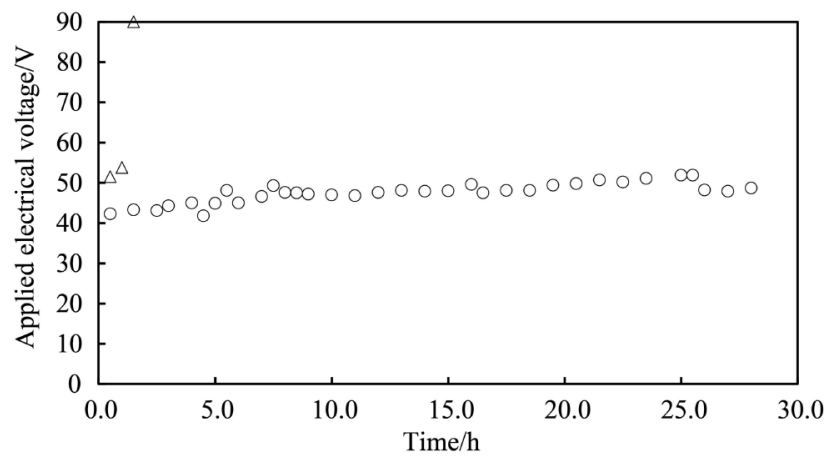

Fig. 6 Effect of FCEMs 1 and 2 on the potential across the electrodes as a function of time: $(\bigcirc)$ both membranes are present and $(\triangle)$ both membranes are absent.

current at a constant potential of $30 \mathrm{~V}$ when the water flow is withheld from each of the three compartments in turn is shown in Fig. 5. The current decreases when the water flow is withheld from the CC. The current increases, and then decreases when the water flow is withheld from the PC. The current increases when the water flow is withheld from the AC. The decrease in the electrical current when water is withheld from the $\mathrm{CC}$ or PC, illustrates that generation of $\mathrm{D}_{\mathrm{H}}$, and $\mathrm{D}_{\mathrm{OH}}$ decreases due to consumption of the water in these compartments. Moreover, the increase in the electrical current when water is withheld from the AC or PC illustrates that the electrical resistance decreases due to concentration of ions at the ICJs. These results verify that $\mathrm{D}_{\mathrm{H}}$ and $\mathrm{D}_{\mathrm{OH}}$ are generated by dissociation of water at WDJs 1 and 2, and that sufficient water must be supplied at the WDJs to achieve continuous water dissociation.

Electrochemical durability of the four-bipolar-boundary device resulting from FCEMs 1 and 2

We determined the extent to which FCEMs 1 and 2 prevented electrochemical degradation of the $\mathrm{AE}$ resin packed in the $\mathrm{AC}$ and $\mathrm{CC}$. When the AE resin is electrolyzed due to oxidation and reduction at the electrodes, electrical current cannot pass between the electrodes, and the applied voltage will increase at a constant current. Variations in the voltage were measured at a constant current of $40 \mathrm{~mA}$ with and without the two membranes FCEMs 1 and 2; the results are shown in Fig. 6. The potential across the electrodes is greater than $90 \mathrm{~V}$ when FCEMs are absent and it is constant when they are present. This result shows that the electrochemical durability of the system increases when FCEMs 1 and 2 are placed on the electrodes. In addition, we confirmed that the system maintained electrochemical stability over six months of continuous use under the same conditions.

Spatially separating the generation of the acid solution from gas evolution

The electrical conductivity of the MSA solution eluted from the four-bipolar-boundary device was measured to confirm that it did not contain gases generated in the system. A well-known characteristic of ECDs is that they produce spike-like noise when a gas is present in the analyte solution. The electrical conductivity of the MSA solution $(\sim 10 \mathrm{mM}, \sim 3840 \mu \mathrm{S} / \mathrm{cm})$ generated by the device is shown in the left panel of Fig. 7. For comparison, the electrical conductivity of the MSA solution $(\sim 10 \mathrm{mM}, \sim 3840 \mu \mathrm{S} / \mathrm{cm})$ produced by $\mathrm{CD}_{\text {boundary0 }}$ is shown in the right panel of Fig. 7. Clearly, the spike-like noise is not 


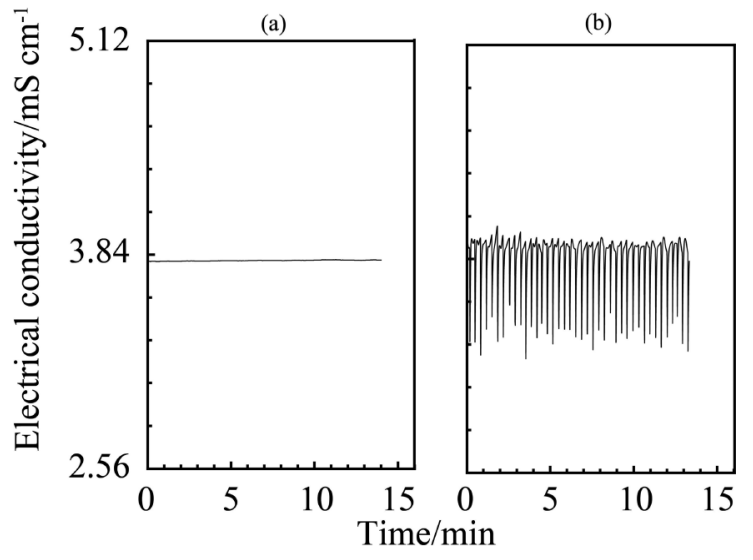

Fig. 7 Electrical conductivity of the MSA solution generated by the four-boundary device and $\mathrm{CD}_{\text {boundary } 0}$ : (a) the four-bipolar-boundary device and (b) $\mathrm{CD}_{\text {boundary }}$.

evident in the effluent for the four-bipolar-boundary device but

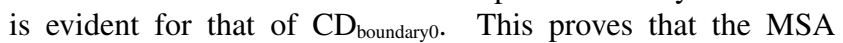
solution produced by the four-bipolar-boundary device is free from gas. We conclude that the four-bipolar-boundary device separates the location where MSA solution is generated from the location where water is electrolyzed.

Performance of the four-bipolar-boundary device: purity of the generated MSA solution

The pressure resistance of the four-bipolar-boundary device was determined. The measurement was performed using an ICA-2000, installing the four-bipolar-boundary device between a pump and an injector. A separation column for cation analysis (CS14) generated a pressure of approximately $9 \mathrm{MPa}$, causing less than $1 \%$ of the water pumped from Inlet 2 to Outlet 2 to leak into the $\mathrm{CC}$ and the AC. Moreover, the membranes in the system did not break during the pressure test. A chromatogram of the standard cations measured by the ICA-2000 is shown in Fig. 8(a). The result illustrates that the four-bipolar-device can be used at a pressure of $9 \mathrm{MPa}$. Furthermore, the concentration of cationic impurities was determined in a sample of the generated MSA solution. Ultra-pure water (without ions) was pumped into the PC of the four-bipolar-boundary device. A chromatogram of the MSA solution $(\sim 10 \mathrm{mM}, 3861 \mu \mathrm{S} / \mathrm{cm}$, $\mathrm{pH} 2.04)$ generated by the four-bipolar-boundary device is shown in Fig. 8(c). For comparison, an MSA solution was prepared by the conventional method $(10 \mathrm{mM}$ MSA prepared from a $1 \mathrm{M}$ MSA stock solution, $3882 \mu \mathrm{S} / \mathrm{cm}, \mathrm{pH} 2.05$ ); the chromatogram is shown in Fig. 8(b). Cations are not present in the MSA solution generated by the four-bipolar-boundary device, whereas they are present in the conventionally prepared solution. This indicates that the cations do not migrate from the $\mathrm{CC}$ and $\mathrm{AC}$ into the PC of the four-bipolar-boundary device. If ultra-pure water is pumped into the $\mathrm{PC}$, the four-bipolar-boundary device can generate a high-purity MSA solution.

Concentration of $\mathrm{HCl}$ solution generated by four-bipolarboundary device

The electrical conductivity and concentration of $\mathrm{HCl}$ solution has been published by The Chemical Society of Japan. ${ }^{20}$ An $\mathrm{HCl}$ solution was generated by pumping $200 \mathrm{mM} \mathrm{NaCl}$ solution (instead of $100 \mathrm{mM}$ MSA solution) into the CC of the four-bipolar-boundary device to examine the relationship between the applied electrical current and the generated acid
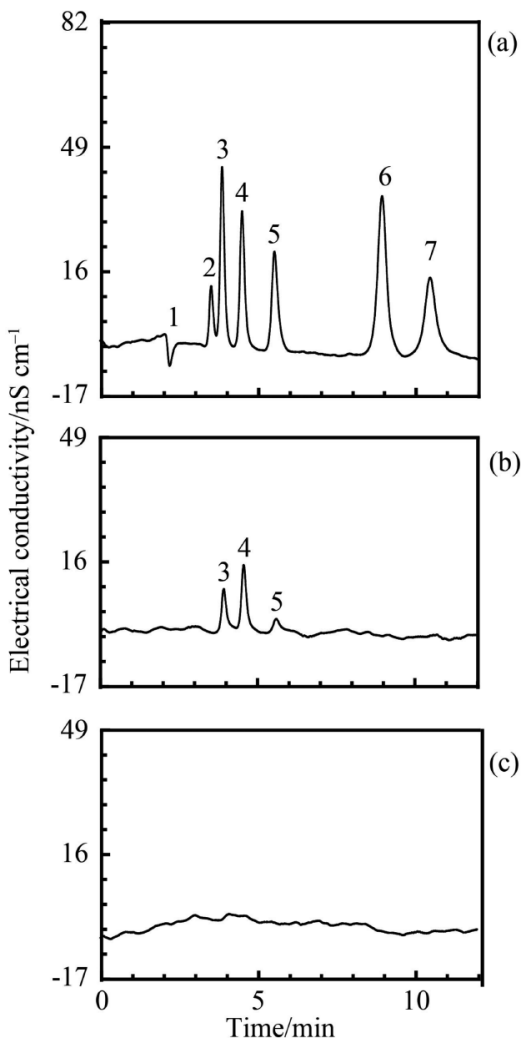

Fig. 8 Electrical conductivity as a function of time to assay cations: (a) standard inorganic cations (1) water dip, (2) $\mathrm{Li}^{+}(5 \mathrm{ppb})$, (3) $\mathrm{Na}^{+}$ (20 ppb), (4) $\mathrm{NH}_{4}^{+}(20 \mathrm{ppb}),(5) \mathrm{K}^{+}(50 \mathrm{ppb}),(6) \mathrm{Mg}^{2+}(50 \mathrm{ppb})$, and (7) $\mathrm{Ca}^{2+}(50 \mathrm{ppb})$; (b) $10 \mathrm{mM}$ MSA solution prepared from $1 \mathrm{M}$ MSA solution stock (3) $\mathrm{Na}^{+}$(4.8 ppb), (4) $\mathrm{NH}_{4}{ }^{+}$(9.2), and (5) $\mathrm{K}^{+}(5.6 \mathrm{ppb}$ ); and (c) $\sim 10 \mathrm{mM}$ MSA solution generated by the four-bipolar-boundary device.

solution concentration. The resulting concentration of $\mathrm{HCl}$ was determined. The concentration of the $\mathrm{HCl}$ solution can be estimated from the electrical conductivity measured by the ECD. The relationship between the $\mathrm{HCl}$ concentration and the applied current was linear $(r=0.999)$, passing through the origin with a slope of $0.566 \mathrm{mM} / \mathrm{mA}$. In a separate experiment, the electrical current applied was fixed at $5 \mathrm{~mA}$, and the electrical conductivity of the purified $\mathrm{HCl}$ solution was measured to be $1186 \mu \mathrm{S} / \mathrm{cm}$, corresponding to an $\mathrm{HCl}$ concentration of $2.84 \mathrm{mM}$ per $1 \mathrm{~mL} / \mathrm{min}$. The electrical current was increased to $10 \mathrm{~mA}$, and the concentration increased to $5.81 \mathrm{mM}$ per $1 \mathrm{~mL} / \mathrm{min}$. This result confirms the linear dependence of the concentration of the $\mathrm{HCl}$ solution on the applied electrical current. We have demonstrated the ability to control the concentration of the $\mathrm{HCl}$ solution using the applied electrical current. The rate of generation of $\mathrm{HCl}$ was $100 \mathrm{mM} \mathrm{HCl}$ per $1 \mathrm{~mL} / \mathrm{min}$ when the maximum applied electrical current (200 mA) was applied to the four-bipolar-boundary device. This result illustrates that acid solution is generated at a rate of $0.01-100 \mathrm{mM}$ per $1 \mathrm{~mL} / \mathrm{min}$ by an applied electrical current $(0-200 \mathrm{~mA})$.

\section{Conclusions}

All the experimental results presented in this paper were consistent with the mechanisms described herein. The four-bipolar-boundary device comprised three compartments 
(AC, PC and CC) separated by two AE membranes; the PC was equipped with two bipolar boundaries that spatially separated the generation of acid solution from the electrolysis of water. Moreover, the $\mathrm{AC}$ and the $\mathrm{CC}$ were equipped with FCEMs 1 and 2 to prevent electrolysis of the AE resin on the electrodes. The four-bipolar-boundary device tolerated high pressures, resisted degradation due to electrolysis at the electrodes, and produced a high-purity acid solution that was free from gases and cationic impurities. The acid solution's concentration was precisely controlled by varying the current applied to the electrodes.

\section{References}

1. SeQuant, "A Practical Guide to Ion Chromatography", 2007, SeQuant AB, Sweden, ISBN 978-91-631-8056-9.

2. D. L. Strong and P. K. Dasgupta, Anal. Chem., 1991, 63, 480.

3. D. L. Strong, C. U. Joung, and P. K. Dasgupta, J. Chromatogr., 1991, 546, 159.

4. H. Small and J. Riviello, Anal. Chem., 1998, 70, 2205.

5. H. Small, Y. Liu, and N. Avdalovic, Anal. Chem., 1998, 70, 3629.

6. B. E. Erickson, Anal. Chem., 1999, 71, 465A.

7. J. S. Fritz and D. T. Gjerde, "Ion Chromatography", 1999, Wiley-VCH, Weinheim.
8. Y. Liu, K. Srinivasan, C. Pohl, and N. Avdalovic, J. Biochem. Biophys. Methods, 2004, 60, 205.

9. Y. Liu, P. Alto, C. A. Pohl, M. J. Mcadams, L. Gatos, H. Small, Z. Lu, M. Liu, and K. Moshfegh, "High Pressure Degas Assembly for Chromatography System and Method", US patent, 2013, 0213225 A1.

10. M. A. Hickner, A. M. Herring, and E. B. Coughlin, J. Polym. Sci. B. Polym. Chem., 2013, 51, 1727.

11. A. Tanioka and K. Shimizu, Bull. Soc. Sea Water Sci. Jpn., 1993, 47, 230.

12. P. Ramirez, H. J. Rapp, S. Mafe, and B. Bauer, J. Electroanal. Chem., 1994, 375, 101.

13. I. C. Bussignana and H. Reiss, J. Membr. Sci., 1983, 15, 27.

14. S. Mafe, J. A. Manzanares, and P. Ramirez, Phys. Rev., 1990, 42, 6245.

15. P. Ramirez, H. J. Rapp, S. Reichle, H. Strathmann, and S. Mafe, J. Appl. Phys., 1992, 72, 259.

16. R. Simons, Electrochim. Acta, 1984, 29, 151.

17. R. Simons, Nature, 1979, $280,824$.

18. H. Masunaga, Y. Higo, M. Ishii, N. Maruyama, and S. Yamazaki, Anal. Sci., 2014, 30, 477.

19. M. C. Sauer, P. F. Southwick, K. S. Spiegler, and M. R. J. Wyllie, Ind. Eng. Chem., 1955, 47, 2187.

20. The Chemical Society of Japan, "The Chemical Handbook Basic Edition", 1993, II-446. 